\title{
Leishmania donovani development in Phlebotomus argentipes: comparison of promastigote- and amastigote-initiated infections
}

\author{
JOVANA SADLOVA ${ }^{1}$, , JITKA MYSKOVA ${ }^{1}$, TEREZA LESTINOVA ${ }^{1}$, JAN VOTYPKA ${ }^{1}$, \\ MATTHEW YEO $^{2}$ and PETR VOLF ${ }^{1}$ \\ ${ }^{1}$ Department of Parasitology, Faculty of Science, Charles University, Vinicna 7, 12844 Prague 2, Czech Republic \\ ${ }^{2}$ Department of Pathogen Molecular Biology, Faculty of Infectious and Tropical Diseases, London School of Hygiene and \\ Tropical Medicine, Keppel Street, WC1E 7HTLondon, UK
}

(Received 2 August 2016; revised 12 October 2016; accepted 15 October 2016; first published online 23 November 2016)

\begin{abstract}
SUMMARY
Leishmania parasites alternate in their life cycle between promastigote stages that develop in the gut of phlebotomine sand flies and amastigotes residing inside phagocytic cells of vertebrate hosts. For experimental infections of sand flies, promastigotes are frequently used as this way of infection is technically easier although ingestion of promastigotes by sand flies is unnatural. Here we aimed to answer a critical question, to what extent do promastigote-initiated experimental infections differ from those initiated with intracellular amastigotes. We performed side-by-side comparison of Leishmania development in Phlebotomus argentipes females infected alternatively with promastigotes from log-phase cultures or amastigotes grown $e x$ vivo in macrophages. Early stage infections showed substantial differences in parasite load and representation of morphological forms. The differences disappeared along the maturation of infections; both groups developed heavy late-stage infections with colonization of the stomodeal valve, uniform representation of infective metacyclics and equal efficiency of transmission. The results showed that studies focusing on early phase of Leishmania development in sand flies should be initiated with intracellular amastigotes. However, the use of promastigote stages for sand fly infections does not alter significantly the final outcome of Leishmania donovani development in P. argentipes and their transmissibility to the vertebrate host.
\end{abstract}

Key words: Leishmania donovani, Phlebotomus, promastigotes, amastigotes, transmission.

\section{INTRODUCTION}

Leishmania parasites (Kinetoplastida: Trypanosomatidae), causative agents of leishmaniases, alternate in their life cycle between intracellular amastigote stages in vertebrate hosts and extracellular promastigote forms in phlebotomine sand flies (Diptera: Psychodidae). In sand flies, Leishmania undergo a complex development through several morphologically and functionally distinct forms (Bates, 2007; Dostalova and Volf, 2012). Sand fly females ingest blood containing macrophages infected with Leishmania amastigotes. In the abdominal midgut, the bloodmeal in engorged flies is encapsulated within the peritrophic matrix, a chitinous envelope secreted by midgut epithelial cells (Lehane, 1997). In this endoperitrophic space, amastigotes transform into procyclic promastigotes that multiply within the bloodmeal. During degeneration of the peritrophic matrix, procyclic promastigotes differentiate into elongated nectomonads, which escape into the ectoperitrophic space (Sadlova and Volf, 2009). Nectomonads first attach to the midgut epithelium,

* Corresponding author: Department of Parasitology, Faculty of Science, Charles University, Vinicna 7, 128 44 Prague 2, Czech Republic. E-mail: sadlovaj@natur. cuni.cz preventing excretion via defecation by the sand fly, later moving anteriorly to the thoracic midgut. Here they give rise to leptomonads which multiply and produce promastigote secretory gel, which fills the thoracic midgut and plays significant role in parasite transmission (Stierhof et al. 1999; Rogers et al. 2002). Finally, leptomonads either attach to the chitin layer of the stomodeal valve and differentiate into haptomonads or transform into small, highly motile infective metacyclics adapted for transmission to the next mammalian host (Sacks and Perkins, 1985; Bates, 2007).

Under laboratory conditions, either Leishmania amastigotes or promastigotes can be used for the initiation of experimental infection in sand flies. While promastigotes can be simply cultivated in vitro and infection is fast and uncomplicated, ingestion of promastigote forms by sand fly females is unnatural. In contrast, amastigote-initiated infections are more natural, but technically difficult and associated with ethical and safety concerns, which make their usage unfavourable. Assessing amastigote dose by direct feeding on infected hosts is difficult and those derived from organs of infected animals requires frequent animal sacrifice and are unavoidably contaminated with host material. Despite cultivation of axenic amastigote-like

Parasitology (2017), 144, 403-410. C Cambridge University Press 2016. This is an Open Access article, distributed under the terms of the Creative Commons Attribution licence (http://creativecommons.org/licenses/by/4.0/), which permits unrestricted re-use, distribution, and reproduction in any medium, provided the original work is properly cited. 
forms being relatively easy for some Leishmania species (Bates, 1993; Gupta et al. 2001), a large number of studies indicate considerable differences when comparing axenic amastigotes with intracellular ones on both genomic and proteomic levels (Holzer et al. 2006; Rochette et al. 2009; Pescher et al. 2011). For these reasons, the cultivation of amastigotes inside macrophages or macrophagelike cell lines is considered the best choice although it is relatively laborious and time consuming (Chang, 1980).

In our study, we aimed to answer the crucial question; to what extent promastigote-initiated experimental infections in sand flies differ from those initiated with amastigotes? If the pattern of development does not differ significantly then infection via promastigotes would be legitimate. Direct comparisons between these two methods are scarce and effects of this factor on transmission potential have not been compared. There is only a single similar study on Leishmania infantum-Lutzomyia longipalpis parasite-vector model (Freitas et al. 2012) which was, however, performed using axenic amastigotes that may substantially differ from intracellular ones due to the loss of important factors during a long-term in vitro culture (Pescher et al. 2011). Here we compared Leishmania donovani infection in its natural vector Phlebotomus argentipes using culture form promastigotes and amastigotes grown ex vivo in bone marrow-derived macrophages (BMM). We published previously (Pruzinova et al. 2015) that the Ethiopian L. donovani strain (GR374) used in the present study develops similarly in both African and Indian vector species Phlebotomus orientalis and Phlebotomus argentipes, respectively. Additionally, for the first time, the transmission potential of parasites to the vertebrate host was assessed which is the most significant marker of successful Leishmania development in the vector.

\section{MATERIALS AND METHODS}

\section{Sand flies and Leishmania}

A laboratory colony of $P$. argentipes (originating from India) was maintained in the insectary of the Charles University in Prague under standard conditions (at $26^{\circ} \mathrm{C}$ fed on $50 \%$ sucrose with a $14 \mathrm{~h}$ light/ $10 \mathrm{~h}$ dark photoperiod) as described previously (Volf and Volfova, 2011).

Amastigote Leishmania stages were grown in BMMs differentiated from precursor cells of BALB/c mice in the presence of L929 fibroblast cell culture supernatant as a source of macrophagecolony stimulating factor (M-CSF). Leishmania donovani (MHOM/ET/2010/GR374) promastigotes transfected with green fluorescence protein as described in Sadlova et al. (2011) were cultured in
M199 medium (Sigma) containing 10\% heat-inactivated fetal calf serum (Gibson) supplemented with 1\% BME vitamins (Basal Medium Eagle, Sigma), $2 \%$ sterile urine, $250 \mu \mathrm{g} \mathrm{mL}^{-1}$ amikacin (Amikin, Bristol-Myers Squibb) and $150 \mu \mathrm{g} \mathrm{mL}^{-1}$ selective antibiotic G 418 (Sigma). Macrophages were exposed to stationary-phase parasites at a parasiteto-macrophage ratio of eight promastigotes to one macrophage. Both infected and uninfected macrophages were cultured in complete RPMI-1640 medium (Sigma) containing 10\% FBS (fetal bovine serum), 20\% L929 cell culture supernatant, 1\% penicillin-streptomycin (Sigma), $2 \mathrm{~mm}$ of L-glutamine (Sigma) and $0.05 \mathrm{~mm}$ of $\beta$-mercapto-ethanol at $37^{\circ} \mathrm{C}$ with $5 \% \mathrm{CO}_{2}$.

\section{Sand fly infections}

To obtain amastigote stages, L. donovani were cocultivated with BMM for $72 \mathrm{~h}$ and non-internalized parasites were removed by washing $3-5$ times with preheated culture medium. Numbers of amastigotes per macrophages were counted by fluorescent microscopy of live macrophages. The infected macrophages were removed from the culture plates using trypsin-EDTA solution (Sigma), centrifuged at $300 \boldsymbol{g}$ for $10 \mathrm{~min}$ and resuspended in heat-inactivated rabbit blood for sand fly infections at the concentration of $10^{6}$ amastigotes $\mathrm{mL}^{-1}$.

For promastigote-initiated infections, promastigotes from log-phase cultures (day 3-4 post-inoculation) were resuspended in heat-inactivated rabbit blood at concentration of $10^{6}$ promastigotes $\mathrm{mL}^{-1}$.

Sand fly females (5-9 days old) were pooled and infected by feeding through a chick-skin membrane either on amastigote- or on promastigote-containing suspension. Engorged sand flies were maintained under standard conditions. Females were dissected at days 1, 2, 4 and 8 post bloodmeal (PBM), and the abundance and location of Leishmania infections in the sand fly digestive tract was examined by fluorescent microscopy. Parasite loads were graded as light ( $<100$ parasites per gut), moderate (100-500 parasites per gut) and heavy ( $>500$ parasites per gut) based on Myskova et al. (2008). Experiments were performed in duplicate.

\section{Transmission by bite and collection of samples for quantitative real-time PCR ( $q P C R)$}

$\mathrm{BALB} / \mathrm{c}$ mice were offered to experimentally infected $P$. argentipes at various time intervals PBM (from days 7-10 PBM). Sand flies (usually 10 females) were placed in a cage $\left(20 \times 20 \mathrm{~cm}^{2}\right)$ and allowed to feed on the single mouse anaesthetized with ketamin/xylazin (150 mg and $15 \mathrm{mg} \mathrm{kg}^{-1}$, respectively) for approximately $1 \mathrm{~h}$. Location of biting of each feeding female was recorded by drawing into the schematic picture of mouse body 
and engorged flies were collected by an aspirator immediately after terminating their bloodmeal. After exposure, mice were sacrificed by injecting them an overdose of ketamin/xylazin and localized tissue excised at the bite location. For sand flies which moved during the feeding, all feeding places were collected into one pooled sample. All samples (skin biopsies and corresponding fed sand flies) were stored at $-20^{\circ} \mathrm{C}$ until DNA extraction for $\mathrm{qPCR}$.

\section{$q P C R$}

Extraction of total DNA from rodent tissues and sand flies was performed using a DNA tissue isolation kit (Roche Diagnostics, Indianapolis, IN) according to the manufacturer's instructions. The qPCR for detection and quantification of Leishmania parasites was performed in Bio-Rad iCycler \& iQ Real-Time PCR Systems using the SYBR Green detection method (iQ SYBR Green Supermix, Bio-Rad, Hercules, CA) as described previously (Myskova et al. 2008) using the kinetoplast primers (forward primer 5'-C'TTTTCTGGTCCTCCGGGTAGG-3' and reverse primer 5'-CCACCCGGCCC'TATTT'TA CACCAA-3') (Mary et al. 2004).

\section{Morphometry of parasites}

Midgut smears of sand flies infected with L. donovani were fixed with methanol, stained with Giemsa, examined by light microscopy with an oil immersion objective and photographed (Olympus DP70). Body length, flagellar length and body width of 120 randomly selected promastigotes from four females/smears were measured for each timepoint PBM and both modes of infection using Image-J software. Four morphological forms were distinguished (Fig. 1), based on the criteria of Sadlova et al. (2010) and Rogers et al. (2002): (a) procyclic promastigotes, $\mathrm{PP}$, flagellum $<$ body length and body length $<14 \mu \mathrm{m}$, present before defecation; (b) elongated nectomonads, EN, body length $\geqslant 14 \mu \mathrm{m}$; (c) metacyclic promastigotes, MP, flagellar length $>2$ times body length and body length $<14 \mu \mathrm{m}$, present post defecation, and (d) short promastigotes, SP, body length $<14 \mu \mathrm{m}$ and flagellar length $\leqslant 2$ times body length. The term 'short promastigotes' is derived from the terminology of Walters (1993) (short nectomonad promastigotes), which is the older synonym of leptomonads (leptomonad promastigotes) proposed by Rogers et al. (2002). Haptomonads were not distinguished in this study as they may remain attached to the gut and can be underrepresented on gut smears.

\section{Statistical analysis}

Differences in intensities of infections, percentage of morphological forms found in infected flies and

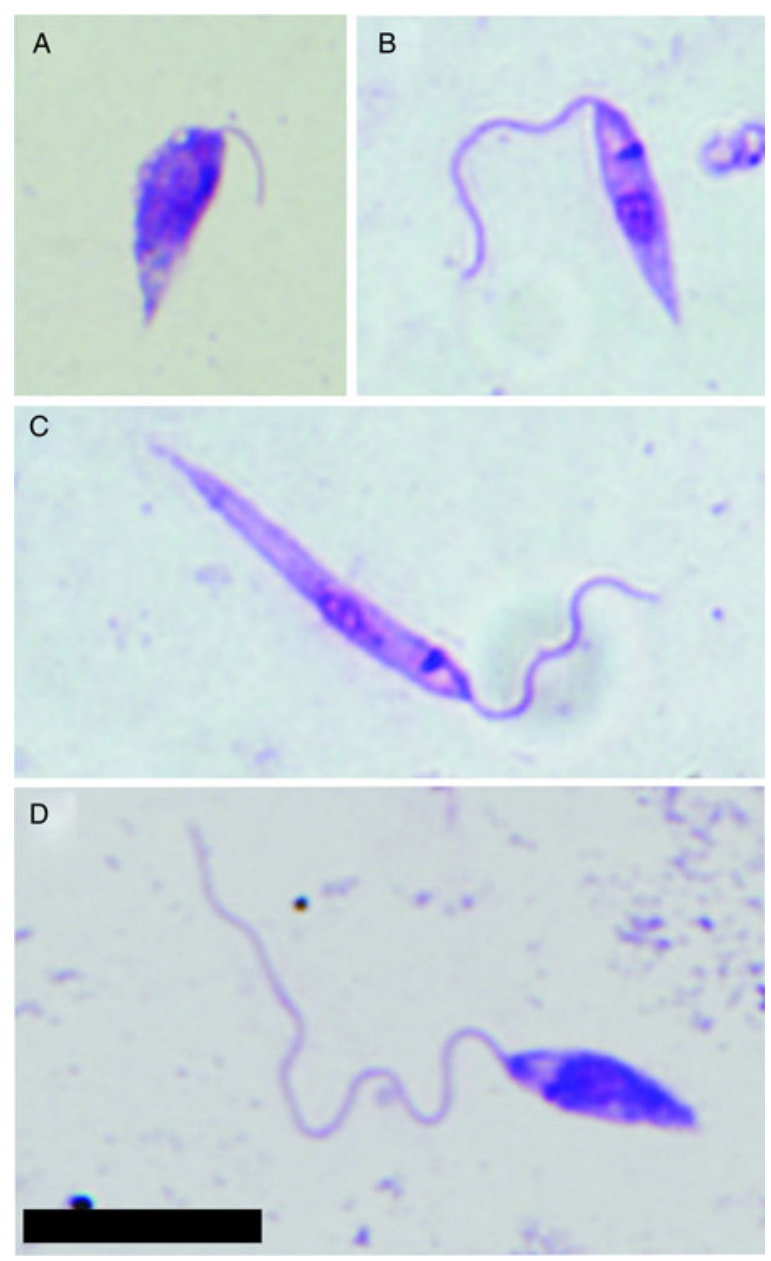

Fig. 1. Morphological forms of L. donovani distinguished in this study based on criteria described in the Materials and Methods section. Parasites from midgut smears of infected sand flies were fixed with methanol and stained with Giemsa. (A) Procyclic promastigote; (B) short promastigote; (C) elongated nectomonad; (D) metacyclic promastigote. Scale bar $=10 \mu \mathrm{m}$.

transmission efficiency were applied to Chi-square $\left(\chi^{2}\right)$ tests. Differences between parasite loads, in sand flies and mice tissues respectively, were tested by non-parametric Mann-Whitney test. All statistical analysis was performed with the statistical software package SPSS version 23.

\section{Animal experimentation guidelines}

Animals were maintained and handled in the animal facility of Charles University in Prague in accordance with institutional guidelines and Czech legislation (Act No. 246/1992 and 359/2012 coll. on Protection of Animals against Cruelty in present statutes at large), which complies with all relevant European Union and international guidelines for experimental animals. Female BALB/c mice were housed in standard plastic T3 cages (Velaz) in groups of six animals with ad libitum access to water and complete feed mixture ST-1 (Velaz). All 


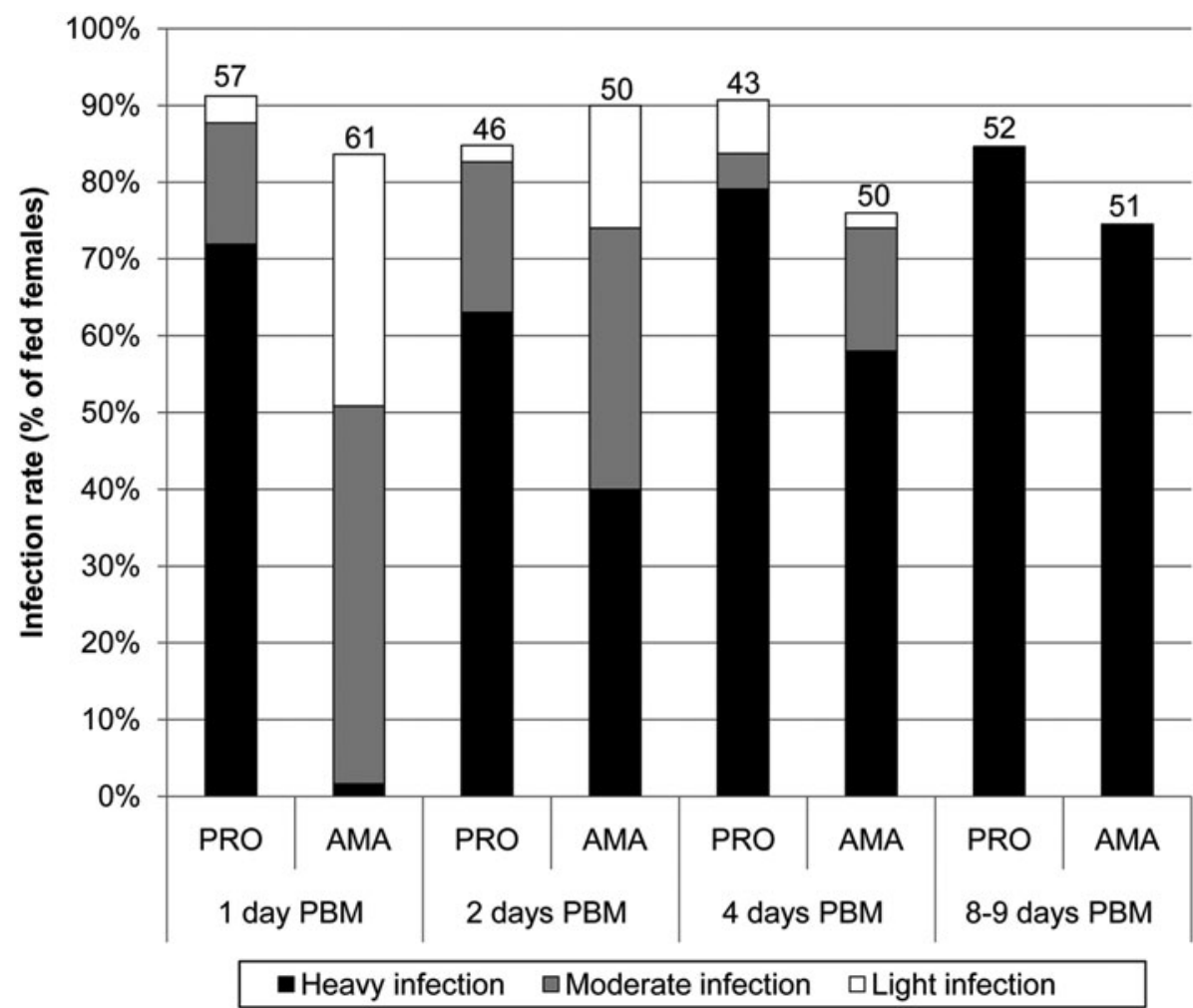

Fig. 2. Infection rates (percent of females infected) and intensities of L. donovani infections in P. argentipes. Parasite load was estimated by fluorescent microscopy: light infections, $<100$ parasites per gut; moderate infections, 100-500 parasites per gut; heavy infections, $>500$ parasites per gut. Numbers of dissected females are shown above bars. PRO, promastigoteinitiated infections; AMA, amastigote-initiated infections; PBM, post bloodmeal. Differences between groups were evaluated using Chi-square test: day 1 PBM, $P<0 \cdot 0001, \chi^{2}=65 \cdot 737$, d.f. $=3$; day 2 PBM, $P=0 \cdot 021, \chi^{2}=9 \cdot 743$, d.f. $=3$; day 4 PBM, $P=0 \cdot 036, \chi^{2}=8 \cdot 518$, d.f. $=3$; days 8-9 PBM, $P=0 \cdot 203, \chi^{2}=1 \cdot 6202$, d.f. $=1$.

the experiments were approved by the Committee on the Ethics of Laboratory Experiments of the Charles University in Prague and were performed under permissions no. MSMT-31114/2013-13 and MSMT-10270/2015-6 of the Ministry of the Environment of the Czech Republic. Investigators are certificated for experimentation with animals by the Ministry of Agriculture of the Czech Republic. Minimum numbers of animals to produce statistically reproducible results were used.

\section{RESULTS}

\section{Development of parasites in $\mathrm{P}$. argentipes females}

During the first four days PBM, infections initiated with amastigote forms of $L$. donovani showed significantly lower intensity than promastigote-initiated ones (Fig. 2). The difference was most pronounced in very early infection, while infections initiated with promastigotes progressed quickly $(72 \%$ of females showed heavy infection by $24 \mathrm{~h}$ PBM), ingested amastigotes underwent a substantial reduction in numbers before parasite load increased (only $2 \%$ of females showed heavy infections at $24 \mathrm{~h} \mathrm{PBM}$ ).

Morphological differences between parasites in both groups were also most significant at $24 \mathrm{~h}$
PBM: procyclic promastigotes were most abundant in amastigote-initiated infections while short promastigotes dominated in promastigote-initiated infections (Fig. 3). By day 2 PBM, increased prevalence of elongated nectomonads in both groups was observed while procyclic forms were still present only in amastigote-initiated infections. Following sand fly defecation by day 4 PBM no substantial morphological differences were detected (Fig. 3).

Interestingly, localization of infections did not differ significantly. In both groups, leishmania development was rapid; parasites escaped from the endoperitrophic space within $24 \mathrm{~h}$ PBM, by $48 \mathrm{~h}$ PBM parasites had reached the thoracic midgut, including cardia region and by day 4 PBM colonized the stomodeal valve in more than $60 \%$ of sand flies in both groups (Figs 4 and 5).

Late-stage development of parasites showed only minor differences between amastigote- and promastigote-initiated infections. All mature infections (by days 8-9 PBM) had heavy parasite load, including colonization of the stomodeal valve in more than $80 \%$ of sand flies in both groups (Figs 2, 4). Elongated nectomonads prevailed in amastigoteinitiated infections, but short promastigotes in promastigote-initiated infections, however differences in presence of metacyclic promastigotes between 


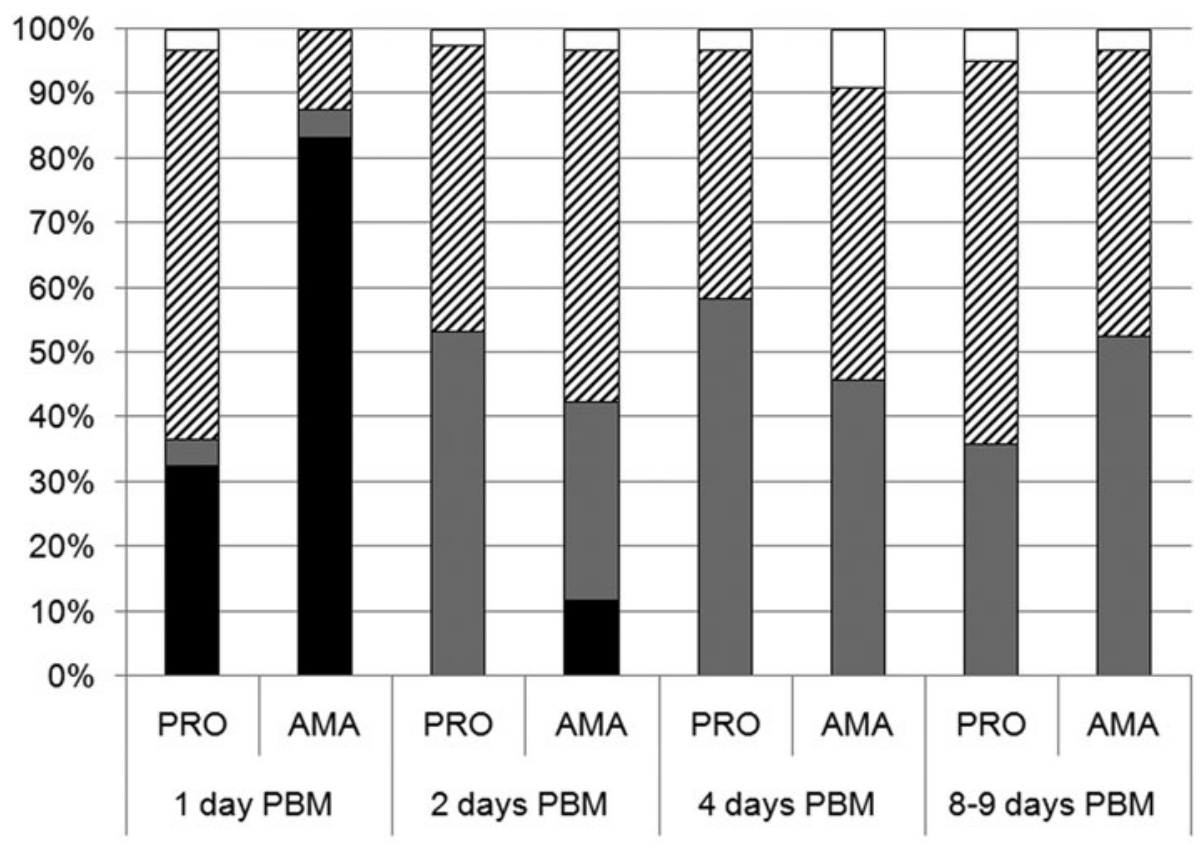

-PP $\square \mathrm{EN} \square \mathrm{SP} \square \mathrm{MP}$

Fig. 3. Morphological forms of $L$. donovani during development in $P$. argentipes. The guts of infected females were sampled at 1, 2, 4 and 8-9 days PBM and parasite morphometry was determined as described in methods. The percentage of each form found in infected flies at each time point is shown. PRO, promastigote-initiated infections; AMA, amastigoteinitiated infections; PBM, post bloodmeal; PP, procyclic promastigotes; EN, elongated nectomonads; SP, short promastigotes; MP, metacyclic promastigotes. Differences between groups were tested by Chi-square test: day $1 \mathrm{PBM}, P$ $<0 \cdot 0001, \chi^{2}=68 \cdot 115$, d.f. $=3$, day 2 PBM, $P<0 \cdot 0001, \chi^{2}=22 \cdot 581$, d.f. $=3$, day 4 PBM, $P=0 \cdot 058, \chi^{2}=5 \cdot 707$, d.f. $=2$, day $8-9$ PBM, $P=0.034, \chi^{2}=6 \cdot 786$, d.f. $=2$.

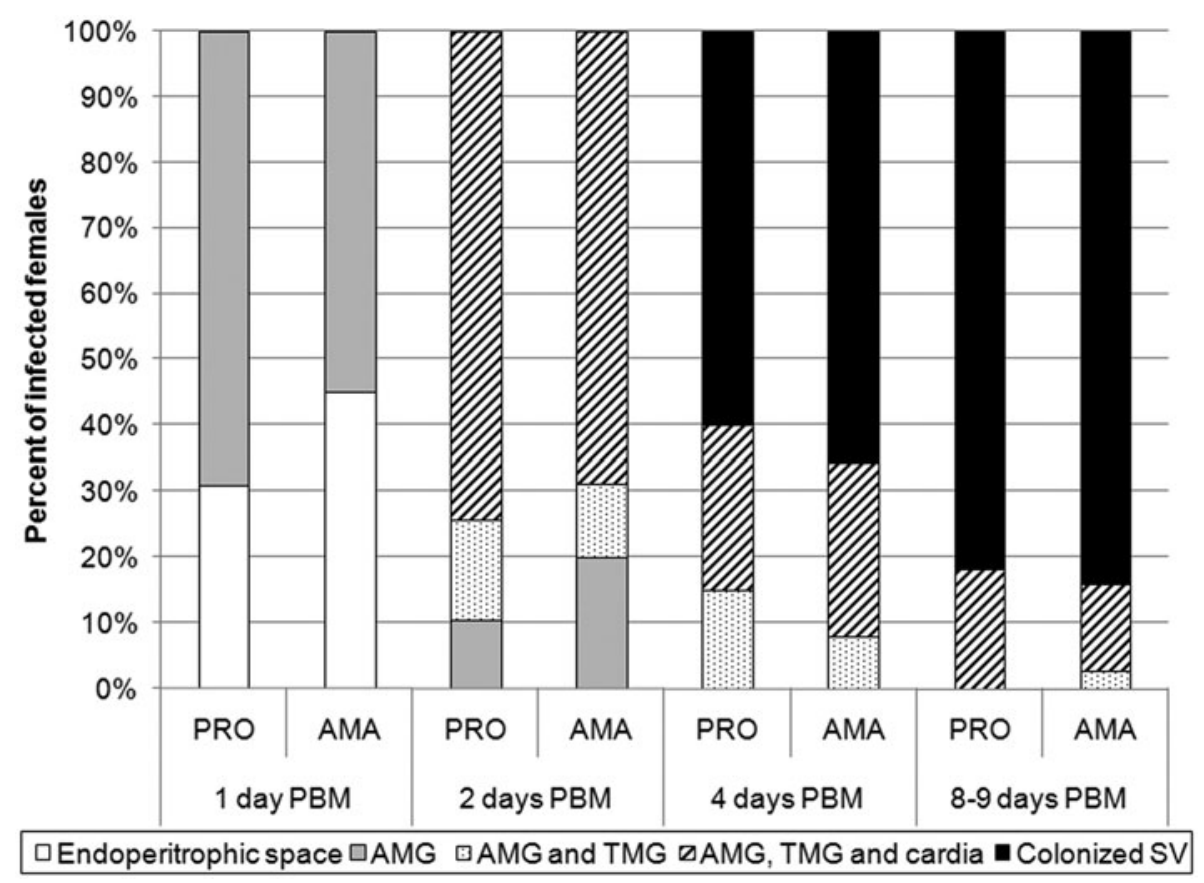

Fig. 4. Location of L. donovani in infected P. argentipes. AMG, abdominal midgut; TMG, thoracic midgut; SV, stomodeal valve. Differences between groups were evaluated using $\chi^{2}$ test: day $1 \mathrm{PBM}, P=0 \cdot 134, \chi^{2}=2 \cdot 247$, d.f. $=1$; day 2 PBM, $P=0 \cdot 436, \chi^{2}=1 \cdot 661$, d.f. $=2$; day 4 PBM, $P=0 \cdot 616, \chi^{2}=0 \cdot 970$, d.f. $=2$; day $8-9$ PBM, $P=0 \cdot 473, \chi^{2}=1 \cdot 497$, d.f. $=2$. 

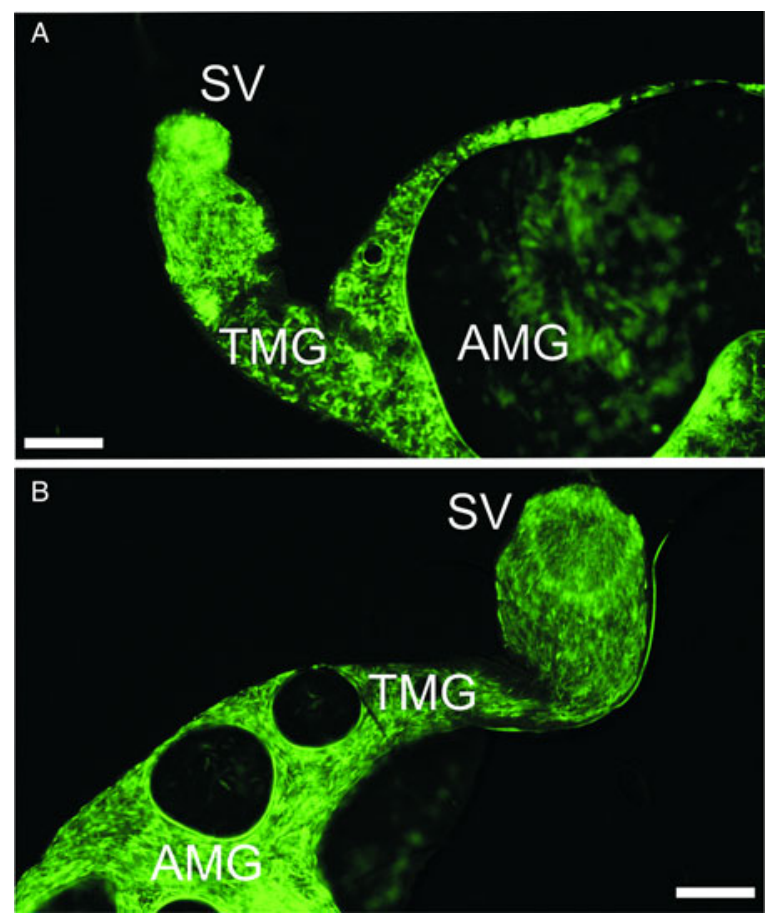

Fig. 5. Images from fluorescent microscope showing amastigote-initiated (A) and promastigote-initiated (B) $L$. donovani infections in $P$. argentipes females by day 4 PBM. Parasites (transfected with GFP) are present in both abdominal and thoracic parts of the midgut and the stomodeal valve is colonized. AMG, abdominal midgut; TMG, thoracic midgut; SV, stomodeal valve. Scale bar $=$ $100 \mu \mathrm{m}$.

the groups were insignificant (Fig. 3; $P=0 \cdot 518$, $\chi^{2}=0 \cdot 417$, d.f. $=1$ ).

\section{Transmission by bite on $B A L B / c$ mice}

The qPCR proved that in both sand fly groups, more than $70 \%$ of females allowed a second feed on mice were infected with Leishmania parasites ('Table 1) and that parasite loads in sand flies did not differ significantly between the groups $(N=117$, MannWhitney $U=1600 \cdot 0, Z=-0 \cdot 369, P=0 \cdot 712)$. From Leishmania-positive flies, 42 and $56 \%$ delivered parasites into the skin of the mice in promastigote- and amastigote-initiated infections, respectively. This apparent difference in transmission efficiency is not statistically significant $\left(P=0 \cdot 270, \chi^{2}=1 \cdot 750\right.$, d.f. $\left.=1\right)$. Numbers of parasites detected in mice tissues by qPCR were slightly higher in the group where sand flies were infected with amastigotes, but not statistically significant (Mann-Whitney $U=138 \cdot 0$, $Z=-1 \cdot 695, P=0 \cdot 090)$.

\section{DISCUSSION}

The results show that mature infections of $L$. donovani in $P$. argentipes initiated with promastigotes did not differ significantly from amastigote initiated infections in the context of parasite load, colonization of the stomodeal valve, presence of infective metacyclic stages and transmission efficiency. In both the compared groups, the development of $L$. donovani was rapid; parasites escaped from the endoperitrophic space before $24 \mathrm{~h}$ PBM and reached the cardia by day $2 \mathrm{PBM}$ with colonization of the stomodeal valve by day $4 \mathrm{PBM}$. This rapid progress is typical for $P$. argentipes and is caused by very fast bloodmeal digestion followed by degradation of the peritrophic matrix and defecation of blood remnants by day 3 PBM (Pruzinova et al. 2015). In contrast there was a pronounced difference between promastigote- and amastigote-initiated infections observed during the first days PBM in both morphology of parasites and kinetics of population growth. Therefore, the use of promastigotes is not recommended for studies on early-stage Leishmania development in sand flies.

As expected, both experimental groups differed in the context of morphological forms in early-stage sand fly infections. While procyclic promastigotes prevailed in amastigote-initiated infection, short promastigotes were predominant in promastigoteinitiated infection. However, elongated nectomonads appeared at the same rate in both groups. Interestingly, localization of infections in sand fly midguts did not differ between the groups, as for example in the rate of parasite escape from the peritrophic matrix into the ectoperitrophic space of the abdominal midgut. The fact that the peritrophic matrix was degraded at the same time in both groups, independently of the prevailing parasite forms present, confirms previous findings on Leishmania major-Phlebotomus duboscqi interactions where a 'sit and wait strategy' was proposed for Leishmania parasites in natural vectors (Sadlova and Volf, 2009) and supports the idea that this process is mediated by sand-fly derived chitinases (Ramalho-Ortigão and Traub-Csekö, 2003; Ramalho-Ortigão et al. 2005) and not substantially influenced by the parasite itself.

In amastigote-initiated infection, substantial reduction of parasite numbers was initially observed, followed by recovery and number increase. The average blood volume ingested by $P$. argentipes females feeding through a chick skin membrane on rabbit blood was $0.6 \mu \mathrm{L}$ (Pruzinova et al. 2015), representing an initial inoculum of about 600 parasites. However, initial numbers of parasites in amastigote-initiated infections were $<500$ in $98 \%$ of females falling to $<100$ in $39 \%$ of females by $24 \mathrm{~h}$ $\mathrm{PBM}(>80 \%$ reduction). The early reduction of parasite numbers in infections initiated with lesionderived amastigotes has been repeatedly described in Leishmania mexicana-infected L. longipalpis (reduction to 88 and $66 \%$ by 6 and 24 h PBM, respectively (Rogers et al. 2002) or reduction to 98-99\% during the first $24 \mathrm{~h} \mathrm{PBM}$ (Rogers et al. 2008) and 
Table 1. Transmission efficiency of Leishmania donovani on BALB/c mice using Phlebotomus argentipes females

\begin{tabular}{|c|c|c|c|c|c|}
\hline \multirow[b]{2}{*}{$\begin{array}{l}\text { Mode of sand fly } \\
\text { infections }\end{array}$} & \multicolumn{3}{|c|}{ Numbers of $P$. argentipes females } & \multirow{2}{*}{$\begin{array}{l}\text { Parasite load in sand flies } \\
\\
\text { Mean } \pm \text { s.D. (median) }\end{array}$} & \multirow{2}{*}{$\begin{array}{l}\begin{array}{l}\text { Transmitted } \\
\text { parasites }\end{array} \\
\begin{array}{l}\text { Mean } \pm \text { s.D. } \\
(\min , \max )\end{array}\end{array}$} \\
\hline & $\begin{array}{l}\text { Feeding } \\
\text { on mice }\end{array}$ & $\begin{array}{l}\text { L. donovani } \\
\text { infected }\end{array}$ & $\begin{array}{l}\text { Transmitting } L \text {. } \\
\text { donovani (transmission } \\
\text { efficiency) }\end{array}$ & & \\
\hline $\begin{array}{l}\text { Promastigote- } \\
\text { initiated }\end{array}$ & 49 & 36 & $15(42 \%)$ & $68090 \pm 8628(59500)$ & $14 \pm 3(1-33)$ \\
\hline $\begin{array}{l}\text { Amastigote- } \\
\text { initiated }\end{array}$ & 68 & 48 & $27(56 \%)$ & $107260 \pm 15672(79100)$ & $58 \pm 21(3-530)$ \\
\hline
\end{tabular}

Parasite numbers were detected using qPCR.

in $L$. major-infected $P$. papatasi [almost $50 \%$ reduction by $4 \mathrm{~h}$ PBM (Pimenta et al. 1997)]. This loss of parasites has been attributed to early attack by digestive proteases in the sand fly gut (Borovsky and Schlein, 1987; Schlein and Jacobson, 1998).

The course of the experimental sand fly infections is influenced with the amount of parasites in the initial infective dose. Although in natural vectors even 1-2 parasites are sufficient for successful establishment of infection (Seblova et al. 2013; Pruzinova et al. 2015) higher infective doses, which are commonly used, are more efficient in producing higher infection rates in sand flies (Anjili et al. 2006; Seblova et al. 2013; Pruzinova et al. 2015). Higher initial doses of $L$. major promastigotes are known to increase parasite loads in $P$. duboscqi and result in a higher percentage of metacyclic forms and increased transmission frequency with more severe pathology in mice (Maia et al. 2011).

Stamper et al. (2011) showed that percentage of metacyclics in sand flies is one of the most important parameters predicting successful Leishmania transmission to the host. In the same study, authors did not find difference in frequency of metacyclics between L. major promastigote- and amastigoteinfected $P$. duboscqi. Accordingly, our experiments with $L$. donovani showed similar numbers of metacyclic forms in mature infections as well as comparable transmission efficiency in amastigote- and promastigote-initiated $P$. argentipes infections. Different results were reported with late stage infections of L. infantum chagasi in L. longipalpis (Freitas et al. 2012). Here metacyclics were 10 times less represented in amastigote-initiated infections compare to promastigote-initiated infection. However, in this case axenic amastigotes were used and efficiency of transmissions on the host was not evaluated. Axenic amastigotes are not adequate alternative to intracellular amastigotes (Holzer et al. 2006; Rochette et al. 2009; Pescher et al. 2011). The study provided by Pescher et al. (2011) revealed important differences in intracellular survival and animal infection of axenic $L$. donovani amastigotes compared with host-derived ones.
Axenic amastigotes differed in cell size and other attributes like expression of the A2 protein representing amastigote-specific marker (Charest and Matlashewski, 1994) responsible for protection of L. donovani from a variety of stresses (McCall and Matlashewski, 2012). Axenic amastigotes were less resistant to nutritional stress compared with splenic-derived ones, which resulted in strong attenuation in establishment of hamster infection (Pescher et al. 2011).

Importantly the transmission efficiency in our experiments was comparable for both modes of infection (42 and 56\% in promastigote and amastigote initiated infections, respectively). Previous experiments with L. infantum suggest that the transmission rate is to a large degree influenced by the parasite species or even strain. Using the same methodologies Maia et al. (2011) demonstrated that 58\% of Phlebotomus perniciosus and 65\% of L. longipalpis transmitted L. infantum CUK3 strain, while only $33 \%$ of $P$. perniciosus and $14 \%$ of L. longipalpis transmitted L. infantum IMT373 strain. Additionally, the number of transmitted parasites has been reported as highly variable among individual females in several Leishmania/vector combinations; females typically transmit tens or hundreds of leishmania parasites but some individuals can deliver several thousand parasites (Warburg \& Schlein, 1986; Kimblin et al. 2008; Maia et al. 2011). In the current study, however, all the females transmitted $<600$ parasites, we did not find individuals transmitting exceptionally high parasite load.

We conclude that regardless of the early decline in abundance of parasites in amastigote initiated infections and initial differences in representation of morphological forms, the $L$. donovani development ultimately results in equivalent pattern of infections in both experimental groups. Following defecation by female sand flies we did not find any significant differences in either course of infections or in the representation of metacyclics. Most importantly, sand flies of both experimental groups transmitted equivalent numbers of parasites to mammalian hosts. In summary, using promastigote stages for 
experimental infections of sand flies does not significantly alter the final outcome of leishmanial development in the vector and can be recommended for this purpose as the most technically convenient and appropriate method. However, for studies concerning early stage infection and development in sand flies (e.g. resistance of Leishmania to proteolytic enzymes or their escape from the peritrophic matrix), intracellular amastigotes are recommended for initiation of infections.

\section{ACKNOWLEDGEMENTS}

We would like to thank Helena Kulikova, Lenka Zitkova and Jana Glogarova for their invaluable technical expertise and administrative support.

\section{FINANCIAL SUPPORT}

This study was funded by Czech Science Foundation GACR (grant number 13-07 500S). J.M. was partially supported by project UNCE 204017/2012.

\section{REFERENCES}

Anjili, C., Langat, B., Lugalia, R., Mwanyumba, P., Ngumbi, P., Mbati, P.A., Githure, J. and Tonui, W. K. (2006). Estimation of the minimum number of Leishmania major amastigotes required for infecting Phlebotomus duboscqi (Diptera: Psychodidae). East African Medical Fournal 83, 68-71.

Bates, P. A. (1993). Axenic culture of Leishmania amastigotes. Parasitology Today 9, 143-146.

Bates, P. A. (2007). Transmission of Leishmania metacyclic promastigotes by phlebotomine sand flies. International Fournal for Parasitology 37, 1097-1106. Borovsky, D. and Schlein, Y. (1987). Trypsin and chymotrypsin-like enzymes of the sandfly Phlebotomus papatasi infected with Leishmania and their possible role in vector competence. Medical and Veterinary Entomology 1, 235-242.

Chang, K. P. (1980). Human cutaneous leishmania in a mouse macrophage line: propagation and isolation of intracellular parasites. Science 209, 1240-1242.

Charest, H. and Matlashewski, G. (1994). Developmental gene expression in Leishmania donovani: differential cloning and analysis of an amastigote-stage-specific gene. Molecular and Cellular Biology 14, 2975-2984.

Dostalova, A. and Volf, P. (2012). Leishmania development in sand flies: parasite-vector interactions overview. Parasites \& Vectors 5, 276.

Freitas, V.C., Parreiras, K.P., Duarte, A.P., Secundino, N. F. and Pimenta, P. F. (2012). Development of Leishmania (Leishmania) infantum chagasi in its natural sandfly vector Lutzomyia longipalpis. The American Fournal of Tropical Medicine and Hygiene 86, 606-612.

Gupta, N., Goyal, N. and Rastogi, A. K. (2001). In vitro cultivation and characterization of axenic amastigotes of Leishmania. Trends in Parasitology 17, 150-153.

Holzer, T. R., McMaster, W. R. and Forney, J. D. (2006). Expression profiling by whole-genome interspecies microarray hybridization reveals differential gene expression in procyclic promastigotes, lesion-derived amastigotes, and axenic amastigotes in Leishmania mexicana. Molecular and Biochemical Parasitology 146, 198-218.

Kimblin, N., Peters, N., Debrabant, A., Secundino, N., Egen, J. Lawyer, P., Fay, M.P., Kamhawi, S. and Sacks, D. (2008). Quantification of the infectious dose of Leishmania major transmitted to the skin by single sand flies. Proceedings of the National Academy of Sciences of the United States of America 105, 10125-10130.

Lehane, M. J. (1997). Peritrophic matrix structure and function. Annual Review of Entomology 42, 525-550.

Maia, C., Seblova, V., Sadlova, J., Votypka, J. and Volf, P. (2011). Experimental transmission of Leishmania infantum by two major vectors: a comparison between a viscerotropic and a dermotropic strain. PLoS Neglected Tropical Disieses 5, e1181

Mary, C., Faraut, F., Lascombe, L. and Dumon, H. (2004) Quantification of Leishmania infantum DNA by a real-time PCR assay with high sensitivity. Fournal of Clinical Microbiology 42, 5249-5255.
McCall, L. I. and Matlashewski, G. (2012). Involvement of the Leishmania donovani virulence factor $\mathrm{A} 2$ in protection against heat and oxidative stress. Experimental Parasitology 132, 109-115.

Myskova, J., Votypka, J. and Volf, P. (2008). Leishmania in sand flies: comparison of quantitative polymerase chain reaction with other techniques to determine the intensity of infection. Fournal of Medical Entomology 45, 133-138.

Pescher, P., Blisnick, T., Bastin, P. and Späth, G. F. (2011) Quantitative proteome profiling informs on phenotypic traits that adapt Leishmania donovani for axenic and intracellular proliferation. Cellular Microbiology 13, 978-991.

Pimenta, P.F., Modi, G. B., Pereira, S. T., Shahabuddin, M. and Sacks, D. L. (1997). A novel role for the peritrophic matrix in protecting Leishmania from the hydrolytic activities of the sand fly midgut. Parasitology 115, 359-369.

Pruzinova, K., Sadlova, J., Seblova, V., Homola, M., Votypka, J. and Volf, P. (2015). Comparison of bloodmeal digestion and the peritrophic matrix in four sand fly species differing in susceptibility to Leishmania donovani. PLoS ONE 10, e0128203.

Ramalho-Ortigão, J. M. and Traub-Csekö, Y. M. (2003). Molecular characterization of Llchit1, a midgut chitinase cDNA from the leishmaniasis vector Lutzomyia longipalpis. Insect Biochemistry and Molecular Biology 33, 279-287.

Ramalho-Ortigão, J. M., Kamhawi, S., Joshi, M. B., Reynoso, D., Lawyer, P. G., Dwyer, D. M., Sacks, D. L. and Valenzuela, J. G. (2005). Characterization of a blood activated chitinolytic system in the midgut of the sand fly vectors Lutzomyia longipalpis and Phlebotomus papatasi. Insect Molecular Biology 14, 703-712.

Rochette, A., Raymond, F., Corbeil, J., Ouellette, M. and Papadopoulou, B. (2009). Whole-genome comparative RNA expression profiling of axenic and intracellular amastigote forms of Leishmania infantum. Molecular and Biochemical Parasitology 165, 32-47.

Rogers, M. E., Chance, M. L. and Bates, P. A. (2002). The role of promastigote secretory gel in the origin and transmission of the infective stage of Leishmania mexicana by the sandfly Lutzomyia longipalpis. Parasitology 124, 495-507.

Rogers, M. E., Hajmova, M., Joshi, M. B., Sadlova, J., Dwyer, D. M., Volf, P. and Bates, P. A. (2008). Leishmania chitinase facilitates colonization of sand fly vectors and enhances transmission to mice. Cellular Microbiology 10, 1363-1372.

Sacks, D. L. and Perkins, P. V. (1985). Development of infective stage Leishmania promastigotes within phlebotomine sand flies. The American Fournal of Tropical Medicine and Hygiene 34, 456-459.

Schlein, Y. and Jacobson, R. L. (1998). Resistance of Phlebotomus papatasi to infection with Leishmania donovani is modulated by components of the infective bloodmeal. Parasitology 117, 467-473.

Seblova, V., Volfova, V., Dvorak, V., Pruzinova, K., Votypka, J., Kassahun, A., Gebre-Michael, T., Hailu, A., Warburg, A. and Volf, P. (2013). Phlebotomus orientalis sand flies from two geographically distant Ethiopian localities: biology, genetic analyses and susceptibility to Leishmania donovani. PLoS Neglected Tropical Diseases 7, e2187.

Stamper, L. W., Patrick, R. L., Fay, M. P., Lawyer, P. G., Elnaiem, D. E., Secundino, N., Debrabant, A., Sacks, D. L. and Peters, N. C. (2011) Infection parameters in the sand fly vector that predict transmission of Leishmania major. PLoS Neglected Tropical Disieses 5, e1288.

Stierhof, Y.D., Bates, P.A., Jacobson, R. L., Rogers, M.E., Schlein, Y., Handman, E. and Ilg, T. (1999). Filamentous proteophosphoglycan secreted by Leishmania promastigotes forms gel-like threedimensional networks that obstruct the digestive tract of infected sandfly vectors. European Fournal of Cell Biology 78, 675-689.

Sadlova, J. and Volf, P. (2009). Peritrophic matrix of Phlebotomus duboscqi and its kinetics during Leishmania major development. Cell and Tissue Research 337, 313-325.

Sadlova, J., Price, H. P., Smith, B. A., Votypka, J., Volf, P. and Smith, D. F. (2010). The stage-regulated HASPB and SHERP proteins are essential for differentiation of the protozoan parasite Leishmania major in its sand fly vector, Phlebotomus papatasi. Cellular Microbiology 12, 1765-1779.

Sadlova, J., Yeo, M., Seblova, V., Lewis, M. D., Mauricio, I., Volf, P. and Miles, M. A. (2011). Visualisation of Leishmania donovani fluorescent hybrids during early stage development in the sand fly vector. PLoS ONE 6, e19851.

Volf, P. and Volfova, V. (2011). Establishment and maintenance of sand fly colonies. Fournal of Vector Ecology 36, S1-S9.

Walters, L. L. (1993). Leishmania differentiation in natural and unnatural sand fly hosts. Fournal of Eukaryotic Microbiology 40, 196-206.

Warburg, A. and Schlein, Y. (1986). The effect of post-bloodmeal nutrition of Phlebotomus papatasi on the transmission of Leishmania major. The American Fournal of Tropical Medicine and Hygiene 35, 926-930. 